\title{
Robustness Analysis of Urban Road Networks from Topological and Operational Perspectives
}

\author{
Wen-Long Shang $\mathbb{D}^{1},{ }^{1}$ Yanyan Chen $\mathbb{D}^{1},{ }^{1}$ Chengcheng Song, ${ }^{1}$ and Washington Y. Ochieng ${ }^{2}$ \\ ${ }^{1}$ Beijing Key Laboratory of Traffic Engineering, College of Metropolitan Transportation, Beijing University of Technology, \\ Beijing 100124, China \\ ${ }^{2}$ Centre for Transport Studies, Imperial College London, London SW7 2AZ, UK \\ Correspondence should be addressed to Wen-Long Shang; shangwl_imperial@bjut.edu.cn
}

Received 23 March 2020; Revised 12 June 2020; Accepted 22 June 2020; Published 14 August 2020

Guest Editor: Chunjia Han

Copyright (c) 2020 Wen-Long Shang et al. This is an open access article distributed under the Creative Commons Attribution License, which permits unrestricted use, distribution, and reproduction in any medium, provided the original work is properly cited.

\begin{abstract}
This study comprehensively analyses the robustness of urban road networks through topological indices based on the complex network theory and operational indices based on traffic assignment theory: User Equilibrium (UE), System Optimum (SO), and Price of Anarchy (POA). Analysing topological indices may pin down the most important nodes for URNs from the perspective of connectivity, while more sophisticated operational indices are helpful to examine the importance of nodes for URNs by taking into account link capacity, travel demand, and drivers' behaviour. The previous way is calculated in a static way, which reduces the computation times and increases the efficiency for quick assessment of the robustness of URNs, while the latter is in a dynamic way, namely, calculating is based on removal of individual nodes, although this way is more likely to capture realistic meanings but consumes huge amount of time. The efforts made in this study try to find the relationship between topological and operational indices so as to assist the assessment of robustness of URNs to local disruptions. Seven realistic urban road networks such as Sioux Falls and Anaheim are used as network examples, and results show that different indices reflect robustness characteristics of urban road networks from different ways, and rank correlations between any two indices are poor although small network such as Sioux Falls have better correlations than others.
\end{abstract}

\section{Introduction}

Traffic road networks and infrastructure are frequently at risk of various types of natural disasters [1], such as the Indonesian tsunami and Hurricane Katrina. In addition, terrorisms are also increasingly rampant, for example, $9 / 11$ attacks in 2001, London bombings in 2005, Paris attacks in 2015, and Brussels bombings in 2016 [2]. No matter what types these disasters are, all of these can cause huge destructions of our infrastructure. Therefore, the robustness of urban road networks has been a central focus of transport planners and managers.

The definitions of robustness of networked systems are presented in different fields. In the IEEE standard computer dictionary [3], robustness is defined as "the degree to which a system or component can function correctly in the presence of invalid inputs or stressful environmental conditions." In the study of electrical networks, Holmgren [4] regards robustness as the ability to keep its structure intact when exposed to perturbations. Immers [5] regard robustness as "the degree to which a system is capable of functioning according to its design specifications in the case of serious disruptions." Boccaletti et al. [6] define robustness as an ability of the network to avoid malfunctioning caused by the damage of a fraction of its constituents. Robustness is always associated with performance of systems [7].

To date, there are many indices and measures for the robustness of networked systems. Some of them measure from the perspective of network topology. Topological indices are all based on the topology of networks without taking into account the distribution of any quantity transported by the networks. These indices include degree 
centrality, clustering coefficient, betweenness centrality, and the size of largest connected component, which are based on the complex network theory. Topological indices can be used to represent the ability the networked systems against failures or attacks due to the fact that these indices are usually related to the robustness of the network and some of these measures are able to reflect the efficiency and functionality of the underlying network [8-11], which are closely associated with the assessment of robustness. The definitions of these topological indices can be found in Section 2 .

Due to the fact that the research related to the robustness assessment based on topological indices is very insufficient, here the studies of other networked systems concerning robustness are summarized. In order to explore the error and attack tolerance of the exponential and scale-free networks, Albert et al. [11] examine the change of diameter, the size of largest cluster, and the average size of the isolated clusters when networks suffer from random failure and targeted attacks. Following this, the network performance of complex networks, which are quantified by the average inverse geodesic length and the size of largest connected subgraph, is studied by Holme [8]. The result shows that removing nodes in a descending order of recalculated degree and betweenness of the current networks is more harmful than removals as the descending order of initial degree and betweenness. Similarly, Crucitti et al. [9] propose mathematical measures based on shortest paths to investigate the global and local efficiency of simulated networks. Afterwards, in order to investigate the cascading effects of electric networks, Crucitti et al. $[12,13]$ propose a measure of global efficiency to examine the performance of networks suffering from removal of nodes. In addition, Crucitti et al. [10] also examine the error and attack tolerance of transportation/ communication networks using efficiency measure. To evaluate the dispersiveness/concentration of road networks in the presence of disasters, Sakakibara et al. [14] propose a topological index, and they define the most robust network as the one that minimizes the isolation of districts when suffering from catastrophic disasters.

Recently, topological analyses of air navigation networks for fifteen countries with a specific focus on betweenness have been conducted by Sun et al. [15]. They point out that a statistical distribution of betweenness of all the nodes in the network serves as an indicator of the network robustness because betweenness represents how frequently a given node lies in the shortest path between any pair of nodes, and more nodes with small betweenness implies that the network is robust against node failures.

The number of research studies on network robustness based on topological indices is considerable, but it is insufficient to capture capacity, drivers' behaviour, and flow patterns of realistic networks, especially for urban road networks.

Compared with the research of robustness from the topology perspective, operational indices, which take into account travel demand, drivers' behaviour, and link capacity, are also employed to access the robustness of networks. A new Network Robust Index (NRI) is proposed by Scott [16] to identify critical links of the network. The NRI is calculated based on the system wide, travel time cost of removing a given link and takes into account network flows, link capacity, and network topology. Following this, a network efficiency measure, which is based on travel disutility from the traffic user equilibrium model, is proposed by Nagurney and Qiang [17] to quantitatively assess the efficiency of congested networks and to rank the links of networks by capturing demand, flows, costs, and user behaviour. Based on this new measure, Nagurney and Qiang [18] utilize the relative change of network efficiency as an indicator to study the robustness of a transportation network while all link capacities decrease gradually. In addition, in order to assess the robustness of networks in the presence of link degradation, a relative total cost index is developed by Nagurney and Qiang [19]. This index is calculated based on total travel cost and is able to capture travel behaviour associated with User Optimization (UO) and System Optimization (SO). In the study, they quantify the robustness by gradually decreasing capacity of all links rather than removing links from the network.

The robustness measures based on operational indices is able to better capture more realistic characteristics of road networks such as travel demand, link capacity, and drivers' behaviour.

The above review suggests that systematic and comprehensive examination of different topological and operational indices is insufficient in the context of network robustness. It seems that the notion of robustness is used somewhat haphazardly. Although each study employs a reasonable network performance index for the certain application, these indices are never assessed on a similar platform and cross-compared with one another. This study aims to address this gap through a comprehensive and systematic comparison and analysis on the main robustness measures of different urban road networks from topological and operational perspectives when such networks are subject to local disruptions. Moreover, we will use Spearman's ranking correlation test to reveal potential correlations among topological indices and proposed operational indices.

The remaining of this paper is organized as follows. Section 2 introduces the methodologies used in this study, including topological indices, such as degree centrality, closeness centrality, and betweenness centrality, and proposed operational indices based on user equilibrium and system optimum principles. Afterwards, a case study including seven urban road networks is presented in Section 3. Conclusions and future works are shown in Section 4.

\section{Methodology}

The methodology used in the study is introduced in this section. The commonly used topological indices arising from the complex network theory, such as degree centrality, average path length, and betweenness centrality, are introduced in detail. Following this, operational indices based on user equilibrium (UE) and system optimum (SO), such as relative total UE and $\mathrm{SO}$ cost, are also presented to assess the robustness of URNs. 


\subsection{Topological Indices of Complex Network Theory}

2.1.1. Degree Centrality. The degree centrality of a node $i$ is the number of links connecting to this node [20] and is formulated as follows:

$$
D_{i}=\sum_{j} b_{i j}
$$

where $b_{i j}$ equals 1 if node $i$ connecting with node $j$ and equals 0 otherwise. This is a seemingly simple yet very important index, as it reflects the node's connectivity and its importance in its vicinity. The degree of a network is the average of the degree of all nodes. A larger network degree implies better connectivity within the network.

2.1.2. Clustering Coefficient. The clustering coefficient of a node $i\left(\mathrm{CC}_{i}\right)$ reflects the local feature and clustering effect of the network. It can be mathematically written as follows:

$$
\mathrm{CC}_{i}=\frac{E_{i}}{k_{i}\left(k_{i}-1\right) / 2}
$$

where $E_{i}$ is the actual number of edges between the neighbours of node $i$ (a node connected with node $i$ is called its neighbour) and $k_{i}$ is the number of the neighbours of node $i[21]$. In social networks, this index tends to measure how close the friends of a given individual are.

2.1.3. Average Path Length. Average path length (APL) is the average length of all shortest paths between any pair of nodes, and it can be shown in the following equation:

$$
\mathrm{APL}=\frac{1}{n(n-1) / 2} \sum_{i>j} d_{i j},
$$

where $d_{i j}$ is the length of the shortest path between $i$ and $j$, if $i$ and $j$ are connected $d_{i j}$ is 1 otherwise 0 . APL, also named characteristic path length, can be employed to quantify the structural characteristics of networks [21]. The diameter of (D) of the network is the maximum $d_{i j}$ for all node pairs. The APL and the $D$ are usually to measure the efficiency and transport performance of the network.

2.1.4. Closeness Centrality. Closeness centrality [22] of a node $i$ is the inverse of the sum of the shortest path distance from node ito other nodes and is normalized by $n-1$, and it is written as follows:

$$
\mathrm{C}_{i}=\frac{n-1}{\sum_{v_{j} \in v, i \neq j} d_{i j}} .
$$

This index is used to measure the accessibility from the node $i$ to other nodes.

2.1.5. Betweenness Centrality. Betweenness centrality $\left(\mathrm{BC}_{i}\right)$ is defined to examine the importance of each node $i$ and can be mathematically expressed as follows:

$$
\mathrm{BC}_{i}=\sum_{j, l \in V} \frac{N_{j l}(i)}{N_{j l}},
$$

where $N_{j l}(i)$ is the number of shortest paths passing through the node $i$ and $N_{j l}$ is the total number of shortest paths between any pair of nodes. The edge weight is the actual distance of the link so it is called weighted betweenness centrality.

2.1.6. Efficiency. This index is first proposed by Crucitti et al. [9] to explore the global efficiency of complex networks and looks very similar with APL, and its formulation is written as follows:

$$
E(G)=\frac{1}{N(N-1)_{-}} \sum_{i \neq j \in G} \frac{1}{d_{i j}} .
$$

The efficiency index $(E)$ is already normalized, that is, $0 \leq E(G) \leq 1$, where $G$ is a directed network. If the nonweighted network is completely connected, $E(G)=1$. In this study, actual distance is assigned to weight of a link. The greater $E$, the better efficiency.

2.2. Operational Indices under Distinct Principles. In this section, we mainly introduce operational indices under user equilibrium and system optimum principles and also review some notations employed in this section. A list of notations used here is summarized in Table 1.

Here, $h_{p}$ and $f_{a}$ are different concepts, but they are closely related to each other. In the network, a link can be passed by one or more paths. Therefore, the link flow $f_{a}$ is related to path flows and can be formulated as follows:

$$
f_{a}=\sum_{p \in P} \delta_{a p} h_{p}, \quad \forall a \in A .
$$

$\delta_{a p}$ is a link-path incident matrix:

$$
\delta_{a p}= \begin{cases}1, & \text { if } a \text { belongs to } p, \\ 0, & \text { if } a \text { does not belong to } p .\end{cases}
$$

Path costs can be presented in the following way:

$$
c_{p}=\sum_{a \in A} \delta_{a p} c_{a}(f), \quad \forall p \in P,
$$

where $c_{a}$ is a unit cost function depending on the link flow $f=\left(f_{a}: a \in A\right)$ and $f_{a}$ denotes flows on each link $a \in A$. Here, we utilize Bureau of Public Roads [23] (BPR) link performance function as the link function:

$$
t\left(f_{a}\right)=t_{a}\left(1+\alpha\left(\frac{f_{a}}{K_{a}}\right)^{\beta}\right), \quad \forall a \in A,
$$

where $t_{a}$ and $K_{a}$ are the free flow travel time and capacity of link $a$, respectively, and $\alpha$ and $\beta$ are positive constants. Due to space limit, the values of all constants in BPR function for all URNs used in the study can be directed to https://github. com/bstabler/TransportationNetworks.

The following flow conservation must hold: 
TABLE 1: List of notations used in Section 2.2.

\begin{tabular}{lc}
\hline Notation & Definition \\
\hline$G(N, A)$ & Represent a directed network, where $N$ is a set of nodes and $A$ is a set of links \\
$W$ & Represent a set of origin-destination $(\mathrm{OD})$ pairs \\
$T_{o s}$ & Represent a fixed travel demand for OD pair $(o, s) \in W$ \\
$P_{o s}$ & Represent a set of paths connecting OD pair $(o, s) \in W$ \\
$c_{p}$ & Represent the unit travel cost over path $p \in P_{o s}$ \\
$h_{p}$ & Represent the flow on path $p$ \\
$f_{a}$ & Represent the flow on link $a$ \\
$\delta_{a p}$ & Represent a link-path incident matrix: Equal 1 if $a \in p$; equals 0 otherwise \\
\hline
\end{tabular}

$$
T_{o s}-\sum_{p \in P_{o s}} h_{p}=0, \quad \forall(o, s) \in W .
$$

Then, the set of feasible flows is presented:

$$
\gamma=\left\{h \geq 0: T_{o s}-\sum_{p \in P_{o s}} h_{p}=0, \quad \forall(o, s) \in W\right\} \text {. }
$$

2.2.1. Relative Total User Equilibrium (UE) Cost When Suffering from Local Disruptions. User Equilibrium (UE) is a principle proposed by Wardrop [24] and assumes that all individual drivers selfishly pursue personal interest maximisation. UE is also widely accepted as a variational inequality (VI) problem. The flow pattern $h^{*}=\left(h_{p}^{*}: p \in P\right)$ is a user equilibrium if and only if

$$
\left.\begin{array}{c}
h^{*} \in \Lambda, \\
\sum_{p \in P} c_{p}\left(h_{p}-h_{p}^{*}\right) \geq 0, \quad \forall h \in \Omega,
\end{array}\right\} \operatorname{VI}(c, \Omega) .
$$

The proof process can be found from [25].

In addition, the UE solution can be obtained from the following mathematical optimization program [26]:

$$
\operatorname{Min} Z=\sum_{a} \int_{0}^{f_{a}} c_{a}\left(f_{a}\right) \mathrm{d} f,
$$

which subjects to

$$
\begin{aligned}
\sum_{p \in P_{o s}} h_{p} & =T_{o s}, \forall(o, s) \in W, \\
f_{a} & =\sum_{o} \sum_{s} \sum_{p} \delta_{a p}^{o s} h_{p}^{o s}, \forall a \in A, \\
h_{p}^{o s} & \geq 0, \forall p \in P_{o s}, \forall(o, s) \in W, \\
f_{a} & \geq 0, \forall a \in A .
\end{aligned}
$$

In this case, the total cost is given by

$$
\mathrm{TC}=\sum_{a \in A} f_{a}^{*} c_{a}\left(f_{a}^{*}\right) .
$$

$\mathrm{TC}_{\mathrm{UE}}$ is denoted as total cost when user equilibrium is reached and can be calculated based on equation (16). Following this, the relative total UE cost against local disruptions are denoted as $\mathrm{RTC}_{\mathrm{UE}}$. This new robustness index is extended based on Nagurney and Qiang [17] and Nagurney and Qiang [19]. $\mathrm{RTC}_{\mathrm{UE}}$ can be presented in the following way:

$$
\mathrm{RTC}_{\mathrm{UE}}^{i}=\frac{\mathrm{TC}_{\mathrm{UE}}^{i}-\mathrm{TC}_{\mathrm{UE}}}{\mathrm{TC}_{\mathrm{UE}}} \times 100 \%,
$$

where $\mathrm{TC}_{\mathrm{UE}}^{i}$ is the total cost under UE principle when node $i$ in the network is removed.

2.2.2. Relative Total System Optimum (SO) Cost When Suffering from Local Disruptions. The second principle of traffic assignment: System Optimum (SO), is also proposed by Wardrop [24]. In contrast to UE principle, SO assumes that there are central controllers to minimize the total cost of the whole road system rather than minimizing individual travellers' travel costs, and how drivers are assigned to routes so as to minimize the total travel costs is described by SO principle. The solution of SO is equivalent to the following nonlinear mathematical optimization program:

$$
\operatorname{Min} Z=\sum_{a} f_{a} c_{a}\left(f_{a}\right)
$$

which subjects to

$$
\begin{aligned}
\sum_{p \in P_{o s}} h_{p} & =T_{o s}, \forall(o, s) \in W \\
f_{a} & =\sum_{o} \sum_{s} \sum_{p} \delta_{a p}^{o s} h_{p}^{o s}, \forall a \in A, \\
h_{p}^{o s} & \geq 0, \forall p \in P_{o s}, \forall(o, s) \in W, \\
f_{a} & \geq 0, \forall a \in A .
\end{aligned}
$$

In this case, $\mathrm{TC}_{\mathrm{SO}}$ is denoted as total cost when system optimum is reached and can be calculated based on equation (16).

Similarly, the relative total SO cost can be denoted as $\mathrm{RTC}_{\mathrm{SO}}^{i}$, which is also extended from Nagurney and Qiang [17]. $\mathrm{RTC}_{\mathrm{SO}}^{i}$ can be calculated by using equation (17):

$$
\mathrm{RTC}_{\mathrm{SO}}^{i}=\frac{\mathrm{TC}_{\mathrm{SO}}^{i}-\mathrm{TC}_{\mathrm{SO}}}{\mathrm{TC}_{\mathrm{SO}}} \times 100 \%
$$

where $\mathrm{TC}_{\mathrm{SO}}^{i}$ is the total cost under SO principle when node $i$ in the network is removed.

2.2.3. Relative Price of Anarchy. Price of anarchy (POA) is proposed to measure the inefficiency of a system caused by selfish behaviour of individuals. The inefficiency of Nash Equilibria is an old idea [27], but Koutsoupias and Papadimitriou [28] first time proposed the term of POA 
in the field of computer science. They utilised the ration between worst-case Nash equilibria to social optimum to measure the inefficiency of a system's lack of coordination [29]. In transport field, all drivers tend to selfishly choose routes so as to minimize their personal travel time, which causes the increase of total travel cost compared to system optimum [30]. In such context, POA is able to effectively quantify the inefficiency of transport networks with selfish routing [31], and it can be mathematically presented as follows:

$$
\rho=\frac{\mathrm{TC}_{\mathrm{UE}}}{\mathrm{TC}_{\mathrm{SO}}} .
$$

This ratio $\rho$ captures the relationship between costs incurred user-oriented behaviour and system-oriented behaviour rule.

According to equation (21), relative price of anarchy (RPOA) can be formulated as follows:

$$
\mathrm{RPOA}^{i}=\frac{\mathrm{TC}_{\mathrm{UE}}^{i}}{\mathrm{TC}_{\mathrm{SO}}^{i}} \times 100 \%
$$

\section{Case Study}

In this study, for the first time, we systematically present the robustness analysis of URNs based on topological operational indices. In this section, Sioux Falls and Anaheim networks are depicted in detail, and other networks such as Tigarten centre, Mitte centre, and Prenzlauburg centre are analysed briefly due to space limit. The calculation of the solutions of UE and SO for large scale networks takes huge amount of computations; hence, only these seven networks are selected (all network data are obtained from https:/github.com/bstabler/ TransportationNetworks). In addition, here we also need to emphasize that local disruptions can be modelled well by removing one node at a time, and this study does not take into account removal of serval nodes at a time because the network performance fluctuates so greatly that the equilibrium is difficult to be achieved.

3.1. Sioux Falls Network. Sioux Falls is located in the east of South Dakoda and has a total area of 190.29 square kilometres [32]. The road networks of Sioux Falls are presented in Figure 1.

The Sioux Falls network consists of 24 nodes and 76 links. Assuming the network is subject to local disruptions, which can be simulated by removing nodes or links here. As equations (17) and (22), the relative total UE cost $\left(\mathrm{RTC}_{\mathrm{UE}}^{i}\right)$, the relative total $\mathrm{SO}$ cost $\left(\mathrm{RTC}_{\mathrm{SO}}^{i}\right)$, and the relative price of anarchy $\left(\mathrm{RPOA}^{i}\right)$ are calculated after individual nodes is removed. Detailed information is summarized in Table 2, and Figure 2 shows the total costs under UE and SO, as well as the POA when individual nodes are removed from the network.

As can be seen from Table 2, topological indices such as degree (D), weighted closeness (WC), clustering coefficient (CC), and weighted betweenness (WBC) centrality are included. These topological indices reflect a certain characteristic of network topology for URNs, which may be used to assess robustness of the networks. Here, Eff denotes efficiency measure (see equation (6)) after individual nodes are removed, and link length in equation (6) is 1 if two nodes are connected otherwise 0 ; while WEff represents weighted efficiency measure when individual nodes are removed, which regards realistic link length as the weight of the links. Both of these indices examine the performance of the network in terms of average path length. The 8 th, 9th, and 10th columns are operational indices: $\mathrm{RTC}_{\mathrm{UE}}^{i}$ and $\mathrm{RTC}_{\mathrm{SO}}^{i}$ are total costs of $\mathrm{UE}$ and $\mathrm{SO}$, respectively, when individual nodes are removed, and $\mathrm{RPOA}^{i}$ is the ratio of $\mathrm{RTC}_{\mathrm{UE}}^{i}$ to $\mathrm{RTC}_{\mathrm{SO}}^{i}$, which assesses the closeness of a UE network state from the system optimum state.

As can be seen from Figure 2, the $\mathrm{RTC}_{\mathrm{UE}}^{i}$ and $\mathrm{RTC}_{\mathrm{SO}}^{i}$ curves have similar tendency, and node $\# 10$ is the most important node in this network, since removing it brings the largest increase in the network-wide cost. Node \#10 happens to have the largest degree in the network. The left graph of Figure 2 shows that node \#10 is located in the central position of the network. However, the $\mathrm{RPOA}^{i}$ presents that node \#2 impacts the network most greatly, though this node is located at the edge of the network.

According to these quantitative topological and operational indices, we rank all the nodes in the network and the results, as shown in Table 3. Among all nine indices, Eff and WEff are ranked in an ascending order, which means nodes with lower efficiency values have the greatest impact on the network. As the rest of the indices, the nodes are ranked in a descending order, that is, nodes that can cause the greatest deterioration of relevant network performance are most important and also require more resources when such disruptions happen.

"-" means these nodes' ranking is meaningless due to the fact that these values are zero, which are caused when the neighbours of a given node in the network are not connected with each other.

Based on Tables 2 and 3, the relationship between those topological and operational indices are analysed as follows.

The spearman correlation method, also named ranking correlation, is used to explore such a relationship. Table 4 depicts the significance of correlation between each pair of indices.

As can be seen from Table $4, D$ has a high negative correlation with nonweighted efficiency index (Eff'), -0.8614 , and also has relatively high correlations with $\mathrm{RPOA}^{i}, \mathrm{RTC}_{\mathrm{UE}}^{i}$, and $\mathrm{RTC}_{\mathrm{SO}}^{i}$. WC has the highest correlation with $D$ and weighed efficiency index, while the correlations between WC and operational indices are not very high. Due to the fact that many nodes have CC being 0 , the correlations between CC and other topological indices are rather low. WBC is used to measure how frequently shortest paths pass through a given node, so weighted efficiency measure has the best correlation with it, and WBC has poor correlations with operational indices. Nonweighted efficiency index has low correlation with $\mathrm{RPOA}^{i}, \mathrm{RTC}_{\mathrm{UE}}^{i}$, and $\mathrm{RTC}_{\mathrm{SO}}^{i}$, and weighted efficiency 


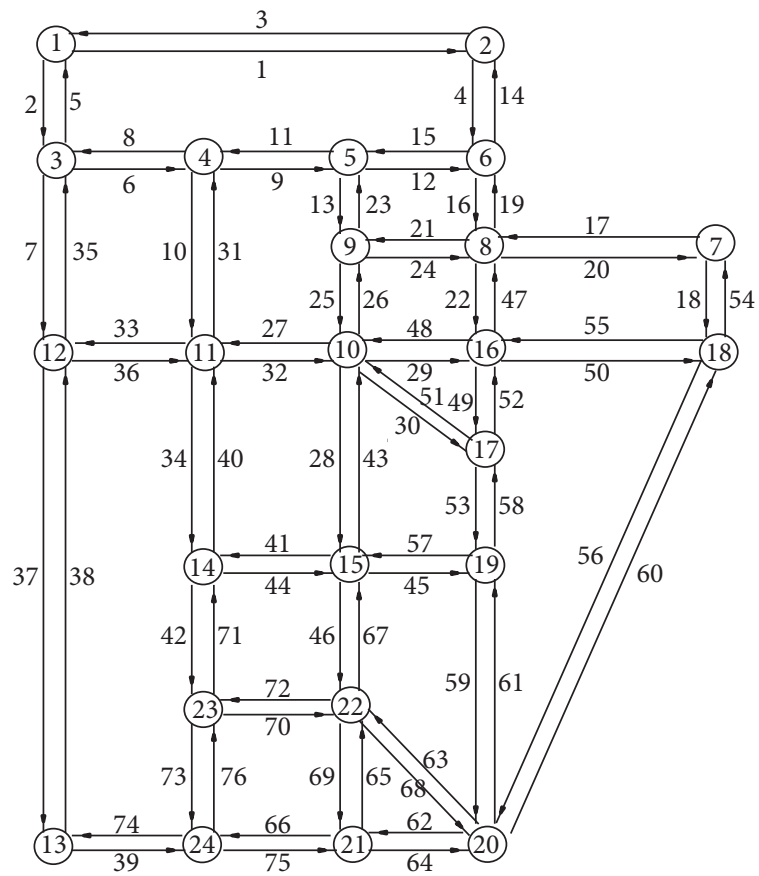

(a)

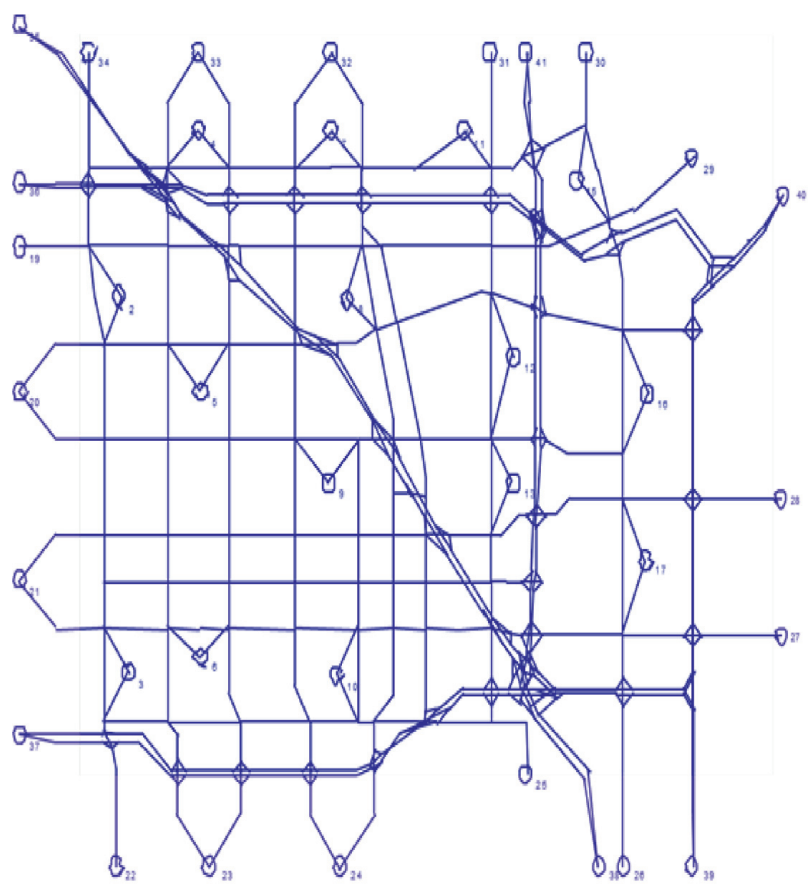

(b)

Figure 1: (a) Sioux Falls network. (b) Anaheim network.

TABle 2: Topological indices and operational indices of all nodes for the Sioux Fall network.

\begin{tabular}{|c|c|c|c|c|c|c|c|c|c|}
\hline \multirow{2}{*}{ Node } & \multicolumn{9}{|c|}{ Indices } \\
\hline & $D$ & WC & $\mathrm{CC}$ & WBC & Eff' & WEff & $\mathrm{RTC}_{\mathrm{UE}}^{i}$ & $\mathrm{RTC}_{\mathrm{SO}}^{i}$ & $\mathrm{RPOA}^{i}$ \\
\hline 1 & 0.0870 & 0.0667 & 0 & 0.0198 & 0.3965 & 0.1117 & 2.3588 & 2.4590 & 1.0088 \\
\hline 2 & 0.0870 & 0.0691 & 0 & 0.0316 & 0.3961 & 0.1116 & 1.0941 & 1.1454 & 1.0140 \\
\hline 3 & 0.1304 & 0.0799 & 0 & 0.1146 & 0.3878 & 0.1078 & 1.1437 & 1.2187 & 1.0038 \\
\hline 4 & 0.1304 & 0.0895 & 0 & 0.1252 & 0.3871 & 0.1067 & 3.3681 & 3.5178 & 1.0044 \\
\hline 5 & 0.1304 & 0.0920 & 0 & 0.1344 & 0.3888 & 0.1060 & 2.0273 & 2.1329 & 1.0039 \\
\hline 6 & 0.1304 & 0.0906 & 0 & 0.1838 & 0.3863 & 0.1044 & 2.3281 & 2.4351 & 1.0065 \\
\hline 7 & 0.0870 & 0.0909 & 0 & 0.0889 & 0.3955 & 0.1071 & 3.3825 & 3.5044 & 1.0107 \\
\hline 8 & 0.1739 & 0.0927 & 0 & 0.1798 & 0.3814 & 0.1041 & 5.0474 & 5.2643 & 1.0029 \\
\hline 9 & 0.1304 & 0.0898 & 0 & 0.0277 & 0.3878 & 0.1096 & 4.7399 & 4.9516 & 1.0019 \\
\hline 10 & 0.2174 & 0.1018 & 0.0400 & 0.1067 & 0.3693 & 0.1062 & 14.3559 & 14.9324 & 1.0013 \\
\hline 11 & 0.1739 & 0.0962 & 0 & 0.0982 & 0.3731 & 0.1085 & 6.7353 & 7.0059 & 1.0037 \\
\hline 12 & 0.1304 & 0.0839 & 0 & 0.1120 & 0.3839 & 0.1080 & 4.1157 & 4.2802 & 1.0065 \\
\hline 13 & 0.0870 & 0.0807 & 0 & 0.0817 & 0.3924 & 0.1086 & 4.0706 & 4.2363 & 1.0060 \\
\hline 14 & 0.1304 & 0.0888 & 0 & 0.0567 & 0.3886 & 0.1091 & 4.2972 & 4.4696 & 1.0061 \\
\hline 15 & 0.1739 & 0.0962 & 0 & 0.1344 & 0.3842 & 0.1060 & 6.3645 & 6.6235 & 1.0036 \\
\hline 16 & 0.1739 & 0.1013 & 0.0667 & 0.1779 & 0.3821 & 0.1033 & 7.5824 & 7.9025 & 1.0015 \\
\hline 17 & 0.1304 & 0.0970 & 0.1333 & 0.1028 & 0.3889 & 0.1053 & 11.7029 & 12.1898 & 1.0005 \\
\hline 18 & 0.1304 & 0.0966 & 0 & 0.1304 & 0.3855 & 0.1053 & 1.8496 & 1.9364 & 1.0081 \\
\hline 19 & 0.1304 & 0.0931 & 0 & 0.0968 & 0.3901 & 0.1056 & 3.6382 & 3.7820 & 1.0076 \\
\hline 20 & 0.1739 & 0.0902 & 0.0652 & 0.0771 & 0.3809 & 0.1083 & 5.3172 & 5.5382 & 1.0037 \\
\hline 21 & 0.1304 & 0.0885 & 0.1305 & 0.1014 & 0.3895 & 0.1076 & 3.1435 & 3.2669 & 1.0088 \\
\hline 22 & 0.1739 & 0.0931 & 0.0652 & 0.1074 & 0.3874 & 0.1060 & 7.5057 & 7.8065 & 1.0034 \\
\hline 23 & 0.1304 & 0.0858 & 0 & 0.0257 & 0.3917 & 0.1080 & 4.4205 & 4.6018 & 1.0052 \\
\hline 24 & 0.1304 & 0.0855 & 0 & 0.1094 & 0.3887 & 0.1057 & 2.3496 & 2.4518 & 1.0081 \\
\hline
\end{tabular}




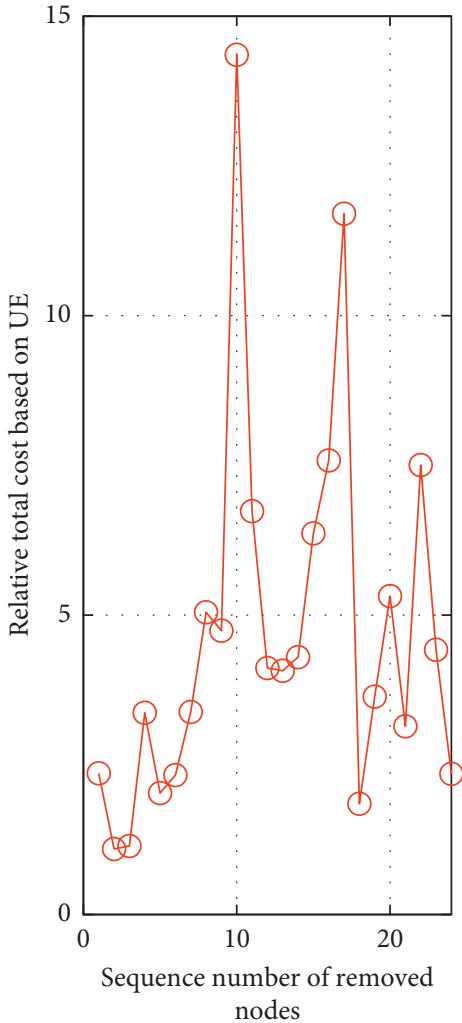

(a)

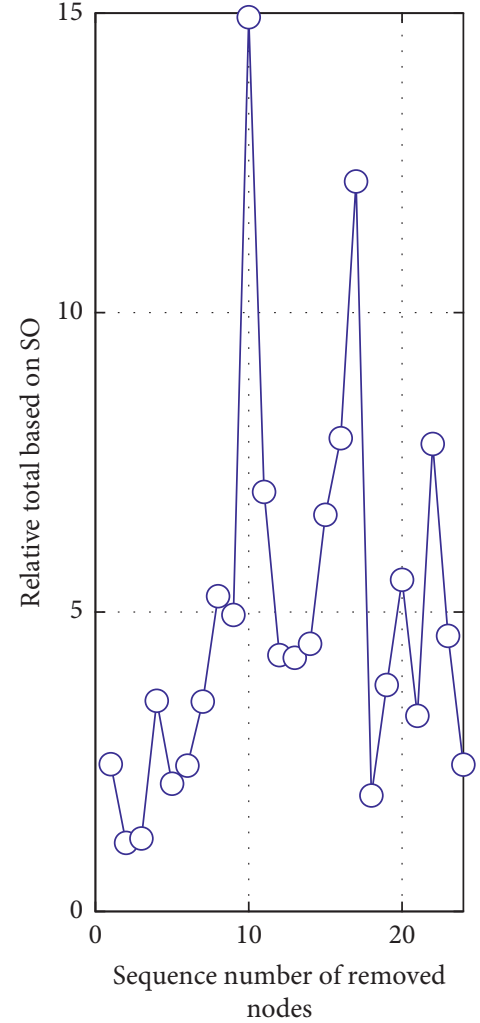

(b)

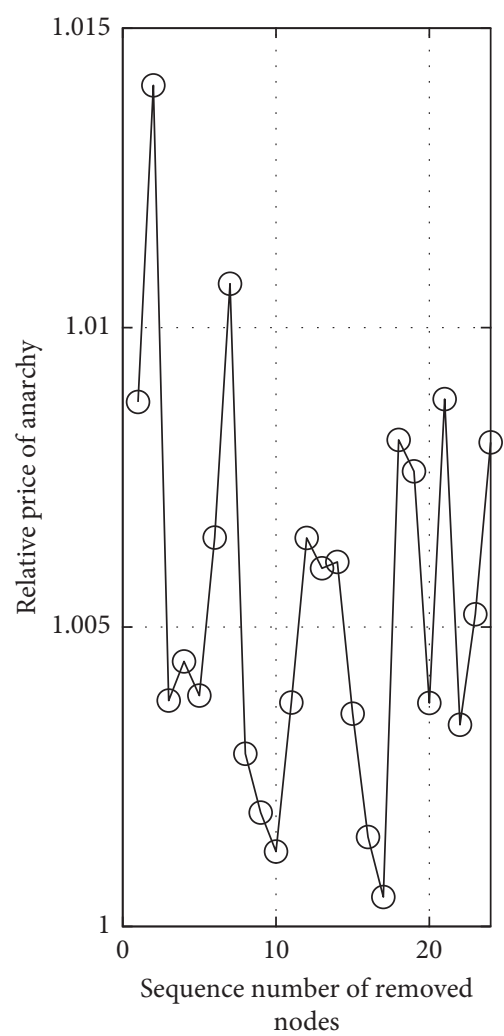

(c)

FIgUre 2: Evolution of $\mathrm{RTC}_{\mathrm{UE}}^{i}$, $\mathrm{RTC}_{\mathrm{SO}}^{i}$, and $\mathrm{RPOA}^{i}$ of Sioux Falls when individual nodes are removed.

index is worse; the correlations between them are $-0.1490,-0.1489$, and -0.2906 , respectively. Based on the above descriptions, we may see that only $D$ index has relatively better correlations with the operational indices compared to the rest of topological indices. The explanation is that the larger the $D$ of a given node implies more road links connected with the node, more likely the removal of the node is to give rise to apparent change of topology structure of the network, so as to cause significant fluctuations of network performance, which is directly related to the operational indices.

3.2. Anaheim Network. Anaheim is located in south east of Downtown Los Angeles, California. The right graph of Figure 1 presents the layout of the Anaheim road network.

As performed for the Sioux Falls network, nine topological and operational indices are calculated to indicate the network performance when suffering from local disruptions. Due to space limitation, only top 20 nodes of these indices are presented. As usual, with the exception of Eff' and WEff', nodes are ranked in a descending order. Table 5 shows the top 20 nodes of these indices for Anaheim network.

Figure 3 depicts how the total UE cost, SO cost, and $\mathrm{RPOA}^{i}$ change as certain node is removed from the network. As can be seen from the evolution of $\mathrm{RTC}_{\mathrm{UE}}^{i}$ and $\mathrm{RTC}_{\mathrm{SO}}^{i}$, node \#62 causes the most deterioration when removed, which highlights its importance, and $\mathrm{RPOA}^{i}$ shows that node \#195 has greatest impact on the price of anarchy. However, it seems that the rankings based on topological indices are not directly related to those based on operational indices.

In order to quantify the ranking correlations between these indices, the Spearman correlation method is employed. Table 6 presents the correlation coefficients between any pair of indices.

As can be seen from Table 6, $\mathrm{RTC}_{\mathrm{UE}}^{i}$ and $\mathrm{RTC}_{\mathrm{SO}}^{i}$ have very poor correlations with topological indices, even with degree, the correlations are just -0.4025 and -0.3819 , respectively. RPOA ${ }^{i}$ has no discernible correlations with these topological indices. In comparison, $\mathrm{RTC}_{\mathrm{UE}}^{i}$ and $\mathrm{RTC}_{\mathrm{SO}}^{i}$ have better correlations with degree, partially due to the homogeneity of the network, that is, nodes with more connections are more important in terms of both topological and operational perspective.

Due to the space limitation, the tables of node rankings and correlation between any pair of indices of other networks such as Tigarten centre, Mitte centre, and Prenzlauburg centre cannot be presented in the main body of this paper, and the detailed information can be shown in Supplementary Material.

Through analysis from ranking correlations of these URNs, there are still no significant correlations between topological and operational indices. However, the correlations between these indices are much better than 
TABLE 3: Ranking of all nodes based on topological indices and operational indices for Sioux Falls.

\begin{tabular}{|c|c|c|c|c|c|c|c|c|c|}
\hline \multirow{2}{*}{ Node } & \multicolumn{9}{|c|}{ Indices } \\
\hline & $D$ & WC & $\mathrm{CC}$ & WBC & $\mathrm{Eff}^{\prime}$ & WEff & $\mathrm{RTC}_{\mathrm{UE}}^{i}$ & $\mathrm{RTC}_{\mathrm{SO}}^{i}$ & $\mathrm{RPOA}^{i}$ \\
\hline 1 & 21 & 24 & - & 24 & 10 & 16 & 18 & 18 & 3 \\
\hline 2 & 22 & 23 & - & 21 & 11 & 8 & 24 & 24 & 1 \\
\hline 3 & 8 & 22 & - & 8 & 20 & 6 & 23 & 23 & 15 \\
\hline 4 & 9 & 15 & - & 7 & 8 & 17 & 16 & 15 & 13 \\
\hline 5 & 10 & 10 & - & 4 & 16 & 18 & 21 & 21 & 14 \\
\hline 6 & 11 & 12 & - & 1 & 12 & 19 & 20 & 20 & 8 \\
\hline 7 & 23 & 11 & - & 17 & 15 & 24 & 15 & 16 & 2 \\
\hline 8 & 2 & 9 & - & 2 & 18 & 5 & 8 & 8 & 20 \\
\hline 9 & 12 & 14 & - & 22 & 6 & 15 & 9 & 9 & 21 \\
\hline 10 & 1 & 1 & 6 & 12 & 4 & 22 & 1 & 1 & 23 \\
\hline 11 & 3 & 5 & - & 15 & 22 & 10 & 5 & 5 & 16 \\
\hline 12 & 13 & 20 & - & 9 & 3 & 4 & 12 & 12 & 9 \\
\hline 13 & 24 & 21 & - & 18 & 9 & 7 & 13 & 13 & 11 \\
\hline 14 & 14 & 16 & - & 20 & 14 & 21 & 11 & 11 & 10 \\
\hline 15 & 4 & 6 & - & 5 & 24 & 3 & 6 & 6 & 18 \\
\hline 16 & 5 & 2 & 3 & 3 & 5 & 12 & 3 & 3 & 22 \\
\hline 17 & 15 & 3 & 1 & 13 & 17 & 23 & 2 & 2 & 24 \\
\hline 18 & 16 & 4 & - & 6 & 21 & 20 & 22 & 22 & 5 \\
\hline 19 & 17 & 7 & - & 16 & 19 & 11 & 14 & 14 & 7 \\
\hline 20 & 6 & 13 & 4 & 19 & 23 & 13 & 7 & 7 & 17 \\
\hline 21 & 18 & 17 & 2 & 14 & 13 & 14 & 17 & 17 & 4 \\
\hline 22 & 7 & 8 & 5 & 11 & 7 & 9 & 4 & 4 & 19 \\
\hline 23 & 19 & 18 & - & 23 & 2 & 2 & 10 & 10 & 12 \\
\hline 24 & 20 & 19 & - & 10 & 1 & 1 & 19 & 19 & 6 \\
\hline
\end{tabular}

'-' means these nodes' ranking is meaningless due to the fact that these 270 values are zero, which are caused by 271 that the neighbours of a given node in the network are not connected with each other.

TABLE 4: Spearman correlations between any indices pairs for Sioux Falls.

\begin{tabular}{lccccccccc}
\hline & $D$ & WC & CC & WBC & Eff & WEff & RTC $_{\text {UE }}^{i}$ & RTC $_{\text {SO }}^{i}$ & RPOA $^{i}$ \\
\hline$D$ & 1 & 0.6762 & 0.4329 & 0.4376 & -0.8614 & -0.4187 & 0.6890 & 0.6972 & -0.7336 \\
WC & 0.6762 & 1 & 0.4199 & 0.4505 & -0.5608 & -0.6612 & 0.6085 & 0.6050 & -0.5910 \\
CC & 0.4329 & 0.4199 & 1 & 0.0469 & -0.2110 & -0.2654 & 0.5415 & 0.5415 & -0.4341 \\
WBC & 0.4376 & 0.4505 & 0.0469 & 1 & -0.5485 & -0.8034 & -0.0283 & -0.0196 & -0.2803 \\
Eff & -0.8614 & -0.5608 & -0.2110 & -0.5485 & 1 & 0.3475 & -0.4966 & -0.5071 & 0.6054 \\
WEff $^{2}$ & -0.4187 & -0.6612 & -0.2654 & -0.8034 & 0.3475 & 1 & -0.1489 & -0.1498 & 0.2906 \\
RTC $_{\text {UE }}^{i}$ & 0.6890 & 0.6085 & 0.5415 & -0.0283 & -0.4966 & -0.1489 & 1 & 0.9991 & -0.7746 \\
RTC $_{\text {UE }}^{i}$ & 0.6972 & 0.6050 & 0.5415 & -0.0196 & -0.5071 & -0.1498 & 0.9991 & 1 \\
RTC $_{\text {UE }}$ & -0.7336 & -0.5910 & -0.4341 & -0.2803 & 0.6054 & 0.2906 & -0.7746 & -0.7842 & -0.7842 \\
\hline
\end{tabular}

those of Sioux Falls and Anaheim. In particular, the average weighted betweenness centrality (WBC) has the largest correlation with $\mathrm{RTC}_{\mathrm{UE}}^{i}$ and $\mathrm{RTC}_{\mathrm{SO}}^{i}$ among all topological indices used in this study, with values are uniformly above 0.63 , and correlations for Mitte and MPF even up to 0.76 and 0.77 and 0.74 and 0.74 , respectively. Therefore, for these networks, although the correlations are still not significant enough, which is expected, WBC seems to be relatively highly related to the total costs under UE and SO principles when networks suffer from local disruptions. In order to confirm this viewpoint, the normalized values of selected four indices for these networks are shown in Figure 4.
As can be seen from Figure 4, WBC has roughly consistent tendency with $\mathrm{RTC}_{\mathrm{UE}}^{i}$ and $\mathrm{RTC}_{\mathrm{SO}}^{i}$ when individual nodes are removed from these networks, though $\mathrm{RTC}_{\mathrm{UE}}^{i}$ and $\mathrm{RTC}_{\mathrm{SO}}^{i}$ in Tiergarten network are less fluctuated than WBC. While Eff seems to have no consistent trend with other indices. The analysis based on Figure 4 confirms the previous viewpoint, that is, $\mathrm{WBC}$ has relatively higher correlations with total costs under UE and SO principles than other indices. A possible explanation is that the nodes that are frequently passed by shortest paths between all O-D pairs carry more flows, so the removal of the nodes with large WBC is more likely to cause the significant increase of total travel times of drivers. 
TABLE 5: Top 20 nodes based on topological and operational indices for Anaheim.

\begin{tabular}{|c|c|c|c|c|c|c|c|c|c|}
\hline \multirow{2}{*}{ Rank } & \multicolumn{9}{|c|}{ Indices } \\
\hline & $D$ & WC & $\mathrm{CC}$ & WBC & Eff & WEff & $\mathrm{RTC}_{\mathrm{UE}}^{i}$ & $\mathrm{RTC}_{\mathrm{SO}}^{i}$ & $\mathrm{RPOA}^{i}$ \\
\hline 1 & 303 & 303 & 151 & 308 & 299 & 308 & 62 & 62 & 195 \\
\hline 2 & 330 & 27 & 152 & 337 & 266 & 337 & 63 & 63 & 194 \\
\hline 3 & 337 & 302 & 224 & 29 & 277 & 29 & 233 & 233 & 193 \\
\hline 4 & 266 & 319 & 225 & 303 & 305 & 303 & 2 & 2 & 187 \\
\hline 5 & 267 & 28 & 241 & 361 & 358 & 304 & 4 & 4 & 188 \\
\hline 6 & 269 & 304 & 242 & 33 & 273 & 299 & 232 & 232 & 189 \\
\hline 7 & 273 & 301 & 245 & 302 & 321 & 302 & 87 & 87 & 190 \\
\hline 8 & 299 & 317 & 246 & 304 & 407 & 307 & 234 & 234 & 191 \\
\hline 9 & 302 & 330 & 334 & 299 & 333 & 361 & 86 & 86 & 192 \\
\hline 10 & 304 & 31 & 228 & 378 & 269 & 28 & 235 & 235 & 140 \\
\hline 11 & 308 & 329 & 229 & 27 & 308 & 267 & 89 & 89 & 141 \\
\hline 12 & 317 & 300 & 239 & 28 & 390 & 294 & 88 & 88 & 204 \\
\hline 13 & 329 & 286 & 240 & 307 & 375 & 306 & 74 & 74 & 203 \\
\hline 14 & 332 & 311 & 222 & 267 & 267 & 269 & 25 & 25 & 236 \\
\hline 15 & 333 & 312 & 223 & 269 & 384 & 300 & 73 & 73 & 196 \\
\hline 16 & 341 & 305 & 226 & 268 & 265 & 305 & 1 & 1 & 198 \\
\hline 17 & 361 & 299 & 227 & 266 & 399 & 330 & 398 & 398 & 197 \\
\hline 18 & 369 & 42 & 243 & 394 & 319 & 378 & 397 & 397 & 142 \\
\hline 19 & 373 & 331 & 244 & 305 & 264 & 394 & 116 & 116 & 143 \\
\hline 20 & 378 & 316 & 247 & 36 & 357 & 33 & 117 & 117 & 231 \\
\hline
\end{tabular}

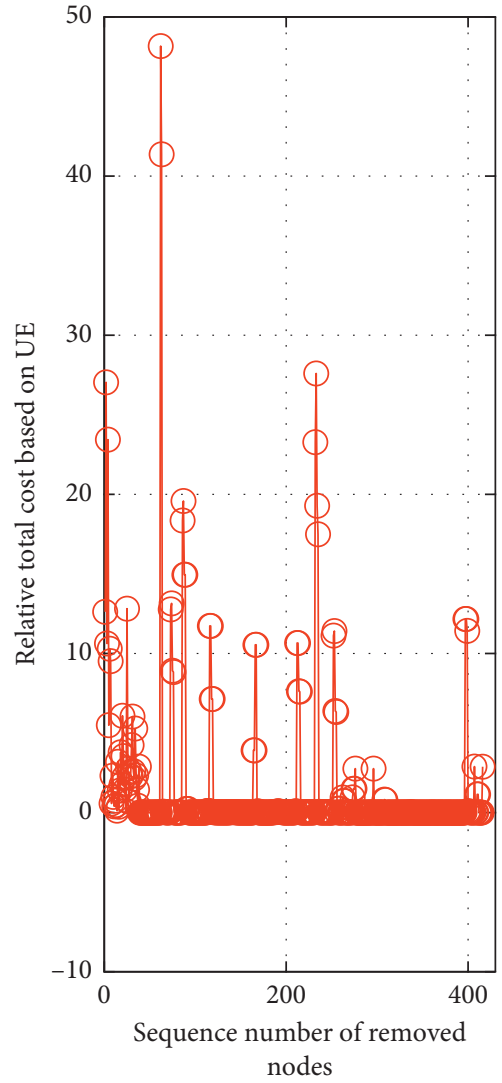

(a)

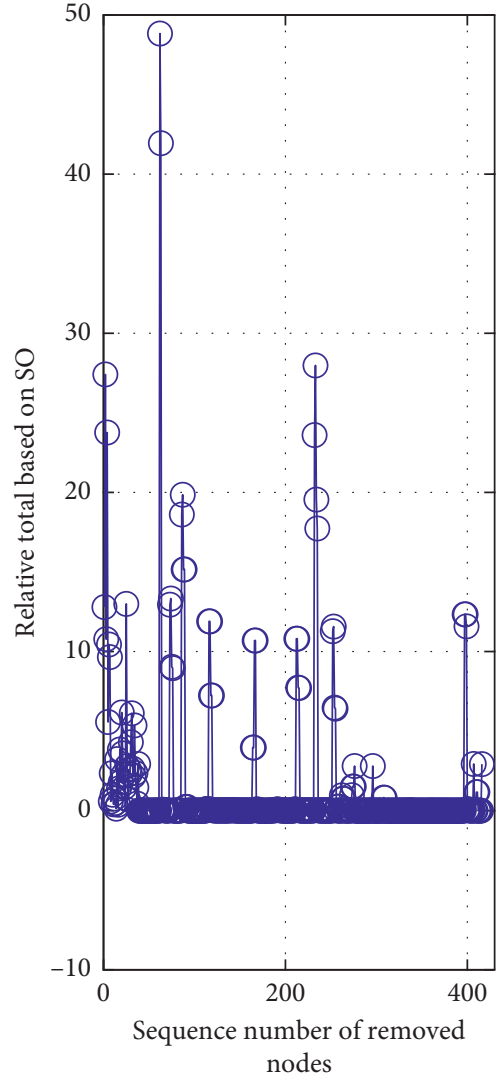

(b)

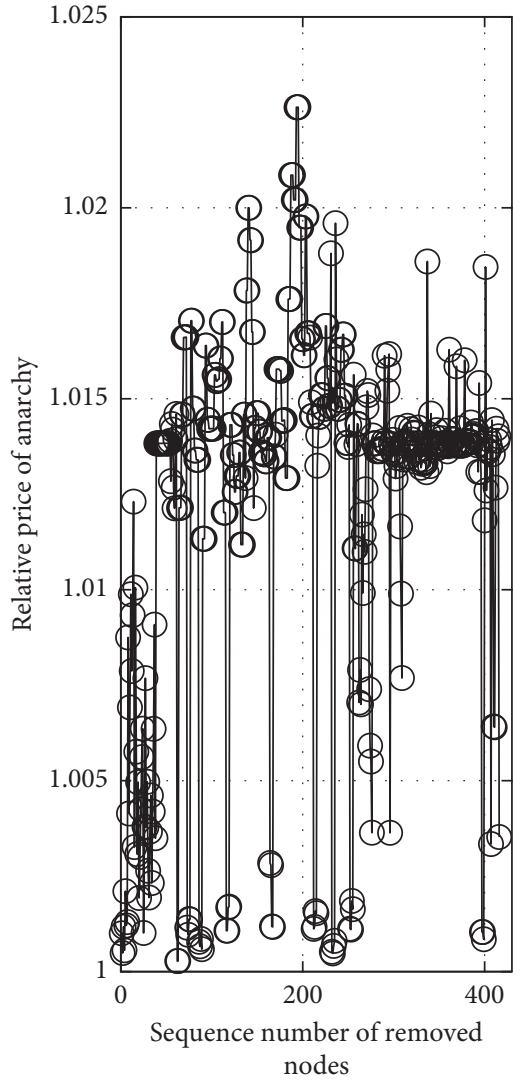

(c)

FIgURE 3: Evolution of $\mathrm{RTC}_{\mathrm{UE}}^{i}, \mathrm{RTC}_{\mathrm{SO}}^{i}$, and $\mathrm{RPOA}^{i}$ of Anaheim when individual nodes are removed. 
TABLE 6: Spearman correlations between any indices pairs for Anaheim.

\begin{tabular}{lccccccccc}
\hline & $D$ & WC & CC & WBC & Eff $^{\prime}$ & WEff $^{\prime}$ & RTC $_{\text {UE }}^{i}$ & RTC $_{\text {SO }}^{i}$ & RPOA $^{i}$ \\
\hline$D$ & 1 & 0.3273 & -0.1363 & 0.5477 & -0.3227 & -0.4445 & -0.4025 & -0.3819 & -0.0272 \\
WC & 0.3273 & 1 & 0.0646 & 0.4808 & -0.2002 & -0.5073 & -0.4737 & -0.4766 & 0.1948 \\
CC & -0.1363 & 0.0646 & 1 & -0.0435 & -0.0988 & -0.0388 & -0.0422 & -0.0533 & 0.3189 \\
WBC & 0.5477 & 0.4808 & -0.0435 & 1 & -0.5208 & -0.7688 & -0.0703 & -0.0341 & -0.0267 \\
Eff & -0.3227 & -0.2002 & -0.0988 & -0.5208 & 1 & 0.4698 & -0.0965 & -0.1008 & 0.0022 \\
WEff $^{i}$ & -0.4445 & -0.5073 & -0.0388 & -0.7688 & 0.4698 & 1 & 0.1025 & 0.0836 & -0.1026 \\
RTC $_{\text {UE }}^{i}$ & -0.4025 & -0.4737 & -0.0422 & -0.0703 & -0.0965 & 0.1025 & 1 & 0.9917 & -0.3260 \\
RTC $_{\text {SO }}^{i}$ & -0.3819 & -0.4766 & -0.0533 & -0.0341 & -0.1008 & 0.0836 & 0.9917 & 1 \\
RPOA $^{i}$ & -0.0272 & 0.1948 & 0.3189 & -0.0267 & 0.0022 & -0.1026 & -0.3260 & -0.3869 & -0.3869 \\
\hline
\end{tabular}

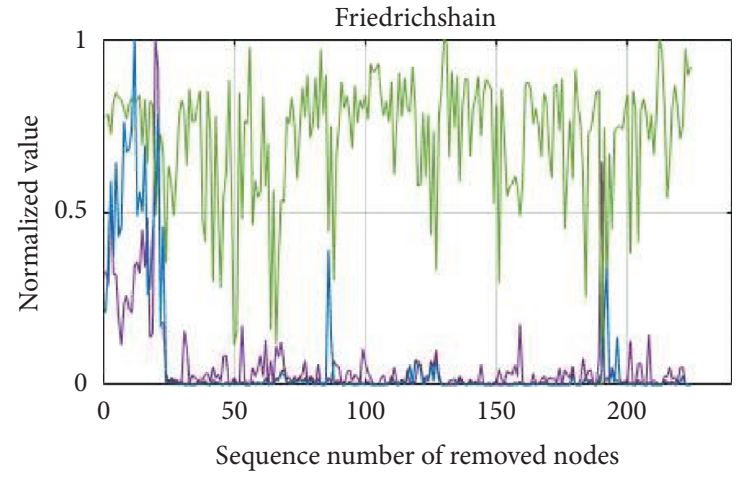

(a)

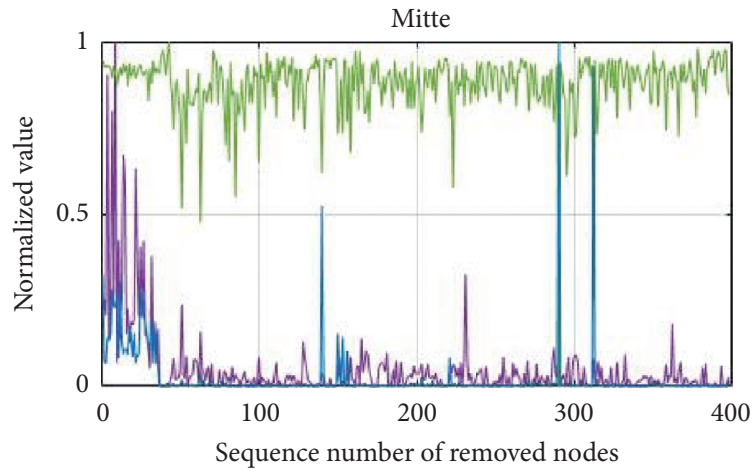

(b)

Figure 4: Continued.

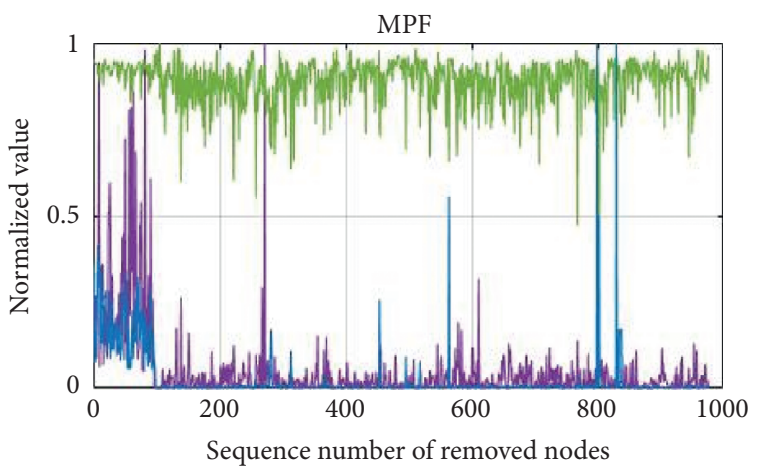

(c)

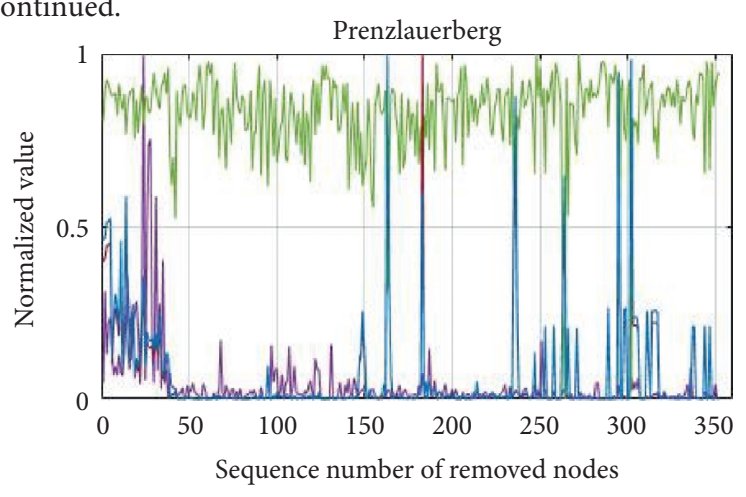

(d)

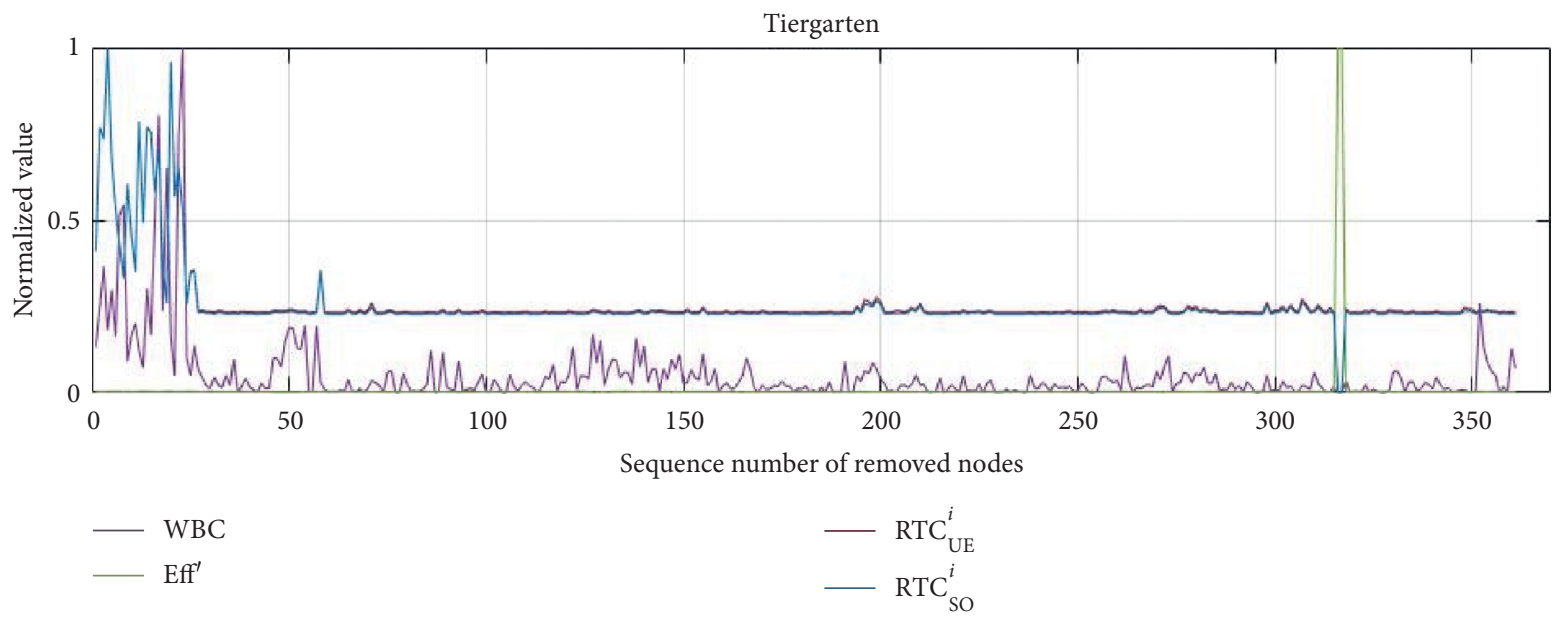

(e)

Figure 4: Normalized values of WBC, Eff', RTC $_{\mathrm{UE}}^{i}$, and RTC $\mathrm{SO}_{\mathrm{SO}}$ for (a) Friedrichshain, (b) Tigarten, (c) Mitte, (d) MPF, and (e) Prenzlauburg suffering from local disruptions. 


\section{Conclusions}

To comprehensively analyse the robustness of URNs suffering from local disruptions, this study utilises topological indices and more sophisticated operational indices (i.e., those based on User Equilibrium (UE), System Optimum (SO), and Price of Anarchy (POA)) to examine the importance of nodes for selected URNs. The topological approach is comparatively more computationally efficient, while the latter takes into account more realistic characteristics and is calculated through certain iterative algorithms, which brings lots of computational overheads. The efforts to seek potential relationships between topological and operational indices have brought huge amount of valuable data for understanding the inherent structural characteristics of the networks:

(1) The results for the Sioux Falls network show that the node (\#10) with the largest degree in the network is also the most important as assessed via user equilibrium $\left(\mathrm{RTC}_{\mathrm{UE}}^{i}\right)$ and system optimum $\left(\mathrm{RTC}_{\mathrm{SO}}^{i}\right)$ solutions. Moreover, degree has relatively high correlations with $\mathrm{RPOA}^{i}, \mathrm{RTC}_{\mathrm{UE}}^{i}$, and $\mathrm{RTC}_{\mathrm{SO}}^{i}$ compared to other topological indices.

(2) The results for Anaheim show that a certain node (\#62), when removed, causes the most deterioration in terms of $\mathrm{RTC}_{\mathrm{UE}}^{i}$ and $\mathrm{RTC}_{\mathrm{SO}}^{i}$ while node \# 195 has greatest impact on the price of anarchy. $\mathrm{RTC}_{\mathrm{UE}}^{i}$ and $\mathrm{RTC}_{\mathrm{SO}}^{i}$ have poor correlations with topological indices, while the correlation between $\mathrm{RPOA}^{i}$ and topological indices is even poorer.

(3) The results for the rest of the selected URNs show higher correlations between topological and operational indices than Sioux Falls and Anaheim. In addition, weighted betweenness centrality (WBC) seems to be relatively highly correlated to the total costs under UE and SO principles when suffering from local disruptions.

Overall speaking, Spearman ranking correlations between topological and operational indices are not significant. However, degree and WBC stand out as the topological indices with relatively higher correlations with operational indices in some individual cases. This study seeks a rapid way of robustness assessment for managers and planners of urban transportation when suffering from local disruptions by exploring potential relationship between topological and operational indices. At this stage, the results of our study just shed light on the possibility and importance of such exploration. In the future, more advanced statistical/machine learning techniques such as deep learning and artificial neural networks can be utilised to provide a more profound understanding of the inherent structural connections of different network indices, thereby bridging the gap between complex network theory and traffic network modelling. In addition, this study only takes into account of removal of one individual node for the robustness assessment, and disequilibrium problems caused by the removal of multiple nodes in sequence may be explored in the future.

\section{Data Availability}

The data of urban road networks used in this study are available from database website https://github.com/bstabler/ TransportationNetwork.

\section{Conflicts of Interest}

The authors declare that there are no conflicts of interest regarding the publication of this paper.

\section{Acknowledgments}

This work was supported in part by the Chinese Scholarship Council (Grant 201308060082) and Key Special Project of Beijing City (Grant Z18110000391801).

\section{Supplementary Materials}

Figure 1: evolution of and of Friedrichshain when nodes are removed. Table 1: the values of top 30 nodes based on topological indices and operational indices for Friedrichshain. Table 2: top 30 nodes based on topological indices and operational indices for Friedrichshain. Table 3: Spearman correlations between any indices pairs for Friedrichshain. Figure 2: evolution of and of Mitte when nodes are removed. Table 4: the values of top 30 nodes based on topological indices and operational indices for Mitte. Table 5: top 30 nodes based on topological indices and operational indices for Mitte. Table 6: Spearman correlations between any indices pairs for Mitte. Figure 3: evolution of MFT when nodes are removed. Table 7: the values of top 30 nodes based on topological indices and operational indices for MPF. Table 8: top 30 nodes based on topological indices and operational indices for MPF. Table 9: Spearman correlations between any indices pairs for MPF. Figure 4: evolution of Prenzlauerberg when nodes are removed. Table 10: the values of top 30 nodes based on topological indices and operational indices for Prenzlauerberg. Table 11: top 30 nodes based on topological indices and operational indices for Prenzlauerberg. Table 12: Spearman correlations between any indices pairs for Prenzlauerberg. Figure 5: evolution of Tiergarten when nodes are removed. Table 13: top 30 nodes based on topological indices and operational indices for Tiergarten. Table 14: top 30 nodes based on topological indices and operational indices for Tiergarten. Table 15: Spearman correlations between any indices pairs for Tiergarten. (Supplementary Materials)

\section{References}

[1] A. Nagurney, "Building resilience into fragile transportation networks in an era of increasing disasters," in Proceedings of the Transportation Research Board 90th Annual Meeting, Washington, DC, USA, January 2011, https://supernet. isenberg.umass.edu/visuals/TRB_Panel_Nagurney_Talk.pdf.

[2] W. L. Shang, Robustness and Resilience Analysis of Urban Road Networks, PhD Thesis, Imperial College, London, UK, 2017.

[3] IEEE, IEEE Standard Computer Dictionary, A Complation of IEEE Standard Computer Glossaries, Institute of Electrical and Electronical Engineers, Piscataway, NJ, USA, 1990. 
[4] Å. Holmgren, "A framework for vulnerability assessment of electric power systems," Critical Infrastructure Advances in Spatial Science, vol. 3155 pages, 2007.

[5] B. Immers, "Reliability and robustness of transportation networks: problem survey and examples," in Proceedings of the NECTAR Cluster Meeting on Reliability of Networks, pp. 19-20, Amsterdam, The Netherlands, December 2004.

[6] S. Boccaletti, V. Latora, Y. Moreno, M. Chavez, and D. Hwang, "Complex networks: structure and dynamics," Physics Reports, vol. 424, no. 4-5, pp. 175-308, 2006.

[7] M. Schillo, "Towards a definition of robustness for marketstyle open multi-agent systems," in Proceedings of the Fifth International Conference on Autonomous Agents, pp. 75-76, New York, NY, USA, June 2011.

[8] P. Holme, "Attack vulnerability of complex networks," Physical Review E, vol. 65, no. 5, 2002.

[9] P. Crucitti, V. Latora, M. Marchiori, and A. Rapisarda, "Efficiency of scale-free networks: error and attack tolerance," Physica A: Statistical Mechanics and Its Applications, vol. 320, pp. 622-642, 2003.

[10] P. Crucitti, "Error and attack tolerance of complex networks," Physica A: Statistical Mechanics and Its Applications, vol. 340, no. 1-3, pp. 388-394, 2004.

[11] R. Albert, H. Jeong, and A.-L. Barabási, "Error and attack tolerance of complex networks," Nature, vol. 406, no. 6794, pp. 378-382, 2000.

[12] P. Crucitti, V. Latora, and M. Marchiori, "Model for cascading failures in complex networks," Physical Review E, vol. 69, no. 4, 2004.

[13] P. Crucitti, V. Latora, and M. Marchiori, "A topological analysis of the Italian electric power grid," Physica A: Statistical Mechanics and Its Applications, vol. 338, no. 1-2, pp. 92-97, 2004

[14] H. Sakakibara, Y. Kajitani, and N. Okada, "Road network robustness for avoiding functional isolation in disasters," Journal of Transportation Engineering, vol. 130, no. 5, pp. 560-567, 2004.

[15] X. Sun, S. Wandelt, and F. Linke, "Topological properties of the air navigation route system using complex network theory," in Proceedings in the 6th International Conference on Research in Air Transportation, Istanbu Technical University, Istanbul, TurkeyIstanbu Technical University, Istanbul, Turkey, May 2014.

[16] D. M. Scott, "Network robustness index-a new method for identifying critical links and evaluating the perfomance of transportation networks center for spatial analysis working paper," 2005.

[17] A. Nagurney and Q. Qiang, "A network efficiency measure for congested networks," Europhysics Letters (EPL), vol. 79, no. 3, p. 38005, 2007.

[18] A. Nagurney and Q. Qiang, "Robustness of transportation networks subject to degradable links," Europhysics Letters (EPL), vol. 80, no. 6, p. 68001, 2007.

[19] A. Nagurney and Q. Qiang, "A relative total cost index for the evaluation of transportation network robustness in the presence of degradable links and alternative travel behavior," International Transactions in Operational Research, vol. 16, no. 1, pp. 49-67, 2009.

[20] A.-L. Barabási and R. Albert, "Emergence of scaling in random networks," Science, vol. 286, no. 5439, pp. 509-512, 1999.

[21] D. J. Watts and S. H. Strogatz, "Collective dynamics of small"world” networks," Nature, vol. 393, no. 6684, pp. 440-442, 1998.

[22] G. Sabidussi, “The centrality index of a graph," Psychometrika, vol. 31, no. 4, pp. 581-603, 1966.
[23] Bureau of Public Roads, Traffic Assignment Manual, U.S. Department of Commerce, Urban Planning Division, Washington, DC, USA, 1964.

[24] J. G. Wardrop, "Road paper. some theoretical aspects of road traffic research," Proceedings of the Institution of Civil Engineers, vol. 1, no. 3, pp. 325-362, 1952.

[25] T. L. Friesz, Dynamic Optimization and Differential Games. International Series in Operations Research \& Management Science, p. 135, Springer US, New York, NY, USA, 2010.

[26] M. Beckmann, C. Mcguire, and C. Winsten, Studies in the Economics of Transportation, Yale University Press, New Haven, CT, USA, 1956, http://cowles.yale.edu/sites/default/files/ files/pub/misc/specpub-beckmann-mcguire-winsten.pdf.

[27] P. Dubey, "Inefficiency of Nash equilibria," Mathematics of Operations Research, vol. 11, no. 1, pp. 1-8, 1986.

[28] E. Koutsoupias and C. Papadimitriou, "Worst-case equilibria," in Proceedings of the 16th Annual Conference on Theoretical Aspects of Computer Science, pp. 404-413, Trier, Germany, March 1999.

[29] S. Robinson, "The price of anarchy," SIAM News, vol. 37, no. 5, 2004.

[30] H. Youn, M. T. Gastner, and H. Jeong, "Price of anarchy in transportation networks: efficiency and optimality control," Physical Review Letters, vol. 101, no. 12, pp. 1-4, 2008.

[31] T. Roughgarden, Selfish Routing and the Price of Anarchy, MIT Press, Cambridge, MA, USA, ISBN 0-262-18243-2, 2005.

[32] https://en.wikipedia.org/wiki/Sioux_Falls,South_Dakota. 